\title{
Electro-optical characterization of IC compatible microcantilevers
}

\author{
A. Pérez-Campos G.F. Iriarte , V. Lebedev, F. Calle
}

\begin{abstract}
The aim of this work is to simulate and optically characterize the piezoelectric performance of CMOS (complementary metal oxide semiconductor) compatible microcantilevers based on aluminium nitride (AIN) and manufactured at room temperature. This study should facilitate the integration of piezoelectric micro-electromechanical systems (MEMS) devices such as microcantilevers, in CMOS technology. Besides compatibility with standard integrated circuit (IC) manufacturing procedures, low temperature processing also translates into higher throughput and, as a consequence, lower manufacturing costs. Thus, the use of the piezoelectric properties of AlN manufactured by reactive sputtering at room temperature is an important step towards the integration of this type of devices within future CMOS technology standards. To assess the reliability of our fabrication process, we have manufactured arrays of free-standing microcantilever beams of variable dimension and studied their piezoelectric performance. The characterization of the first out-of-plane modes of aluminium nitride-actuated piezoelectric microcantilevers has been carried out by using two optical techniques: Laser Doppler Vibrometry and White Light- Interferometry. In order to actuate the cantilevers, a periodic chirp signal in certain frequency ranges was applied between the electrodes of the devices. The nature of the different vibration
\end{abstract}


modes detected has been studied and compared with that obtained by a finite element model based simulation (Comsol Multiphysics), showing flexural as well as torsional modes. The correspondence between theoretical and experimental data is reasonably good, probing the viability of this high throughput and CMOS compatible fabrication process.

To complete the study, X-Ray diffraction as well as $d_{33}$ piezoelectric coefficient measurements were also carried out. 


\section{Introduction}

Micro-electro-mechanical systems (MEMS) comprise an emerging platform for many different applications, such as telecommunication, biosensing, or automotive. Among the different type of MEMS, the use of microcantilevers is widely spread (Vashist, 2007). In the case of resonant microcantilevers, the out-of-plane vibration modes, such as flexural and torsional modes, are the most common ones (Lachut \& Sader, 2007). On the other hand, in-plane modes, which are expected to have better performance in terms of quality factor and mass sensitivity in liquid media (Beardslee et al., 2010) (Ruiz et al., 2012), have been studied to a lesser extent.

The modular integration of MEMS with driving, controlling, and signal-processing CMOS devices is advantageous for improving performance, lowering manufacturing cost, and allowing for separate development of micromechanic and microelectronic technology components. A standard polycrystalline-silicon (poly-Si) based MEMS process typically employs deposition temperatures near $600^{\circ} \mathrm{C}$ and annealing temperatures at or above $900^{\circ} \mathrm{C}$ in order to achieve low-resistivity and low-tensile-stress structural films (Franke et al., 1999a). These temperatures are too high to be compatible with conventional IC metallization schemes, what converts heat generation in one of today's major yield killers (Franke et al., 1999b). On the other hand, in active MEMS, a piezoelectric material is needed, and the usual synthesis temperatures of most commonly used piezoelectric materials $\left(300^{\circ} \mathrm{C}\right.$ of bismuth titanate $\mathrm{Bi}_{4} \mathrm{Ti}_{3} \mathrm{O}_{12}$ (Yahaya \& Salleh, 2004), $700^{\circ} \mathrm{C}$ of lithium niobate $\mathrm{LiNbO}_{3}$ (Lu et al., 2011), between $900^{\circ} \mathrm{C}$ and $1250^{\circ} \mathrm{C}$ of PZT (P. Gr. Lucuta, Fl. Constantinescu, 1985), and around $1000^{\circ} \mathrm{C}$ of lithium tantalite $\mathrm{LiTaO}_{3}$ (Zenin, Lysikov, Nersesyan, Kuznetsov, \& Merzhanov, 1993)) do not allow their integration into CMOS technology. 
Microantilevers are used in a wide variety of applications such as sensors, detectors or energy producers (Wu et al., 2001) (Pei, Tian, \& Thundat, 2004) (Choi, Jeon, Jeong, Sood, \& Kim, 2006) (Oliver et al., 2012). Among the different device configurations for cantilever-based sensors, the use of a piezoelectric layer, embedded between two metal electrodes, is a very attractive alternative. Zinc oxide $(\mathrm{ZnO})$ and lead zirconium titanate (PZT) are piezoelectric materials commonly used in this kind of applications, but they pose a contamination risk in tools shared with CMOS fabrication processes and can be difficult to etch (e.g. low resistivity, composition control, cracking) (Doll, Petzold, Ninan, Mullapudi, \& Pruitt, 2010). In contrast, aluminum nitride (AlN) is a piezoelectric material that is CMOS compatible since it can be grown at room temperature by reactive sputtering. While the $d_{33}$ piezoelectric response of $A I N$ is lower than that of $\mathrm{ZnO}$ or PZT, other material properties (e.g. high elastic modulus and thermal conductivity, low density) make it ideal for many applications (Iriarte, 2003).

In this work we have fabricated piezoelectric microcantilevers and measured them by two optical techniques: aser oppler-ffect ibrometry (LDV) and white light interferometry (WLI). To complete the analysis, natural resonant frequencies were calculated using the finite element modeling (FEM) approach of the Comsol Multiphysics engineering simulation software ("COMSOL Multiphysics Modeling Software," n.d.). A comparison between the six resonant frequencies detected and FEM simulations is done for direct evaluation purposes. X-ray rocking curves as well as the piezoelectric coefficient were also measured as a previous study to approach the piezoelectric behaviour of the structure. 


\section{Experimental}

In this study, we have developed high quality and reliable piezoelectric (AlN-driven) cantilevers for MEMS applications. Each cantilever consists of a thin film of AlN sandwiched between two metal (chromium, $\mathrm{Cr}$ ) electrodes manufactured on top of a silicon (Si-100) wafer (see Figure 1).

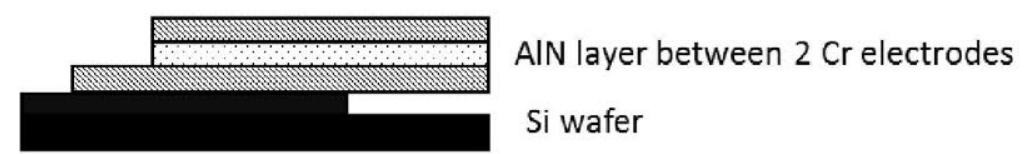

Figure 1: Side view schematic of our AlN driven microcantilevers sandwich structure

An array of microcantilevers with different geometrical dimensions has been studied. The microcantilevers size ranges from $20 \mu \mathrm{m}$ to $50 \mu \mathrm{m}$ in width and $30 \mu \mathrm{m}$ to $625 \mu \mathrm{m}$ in length (see Figure 2).

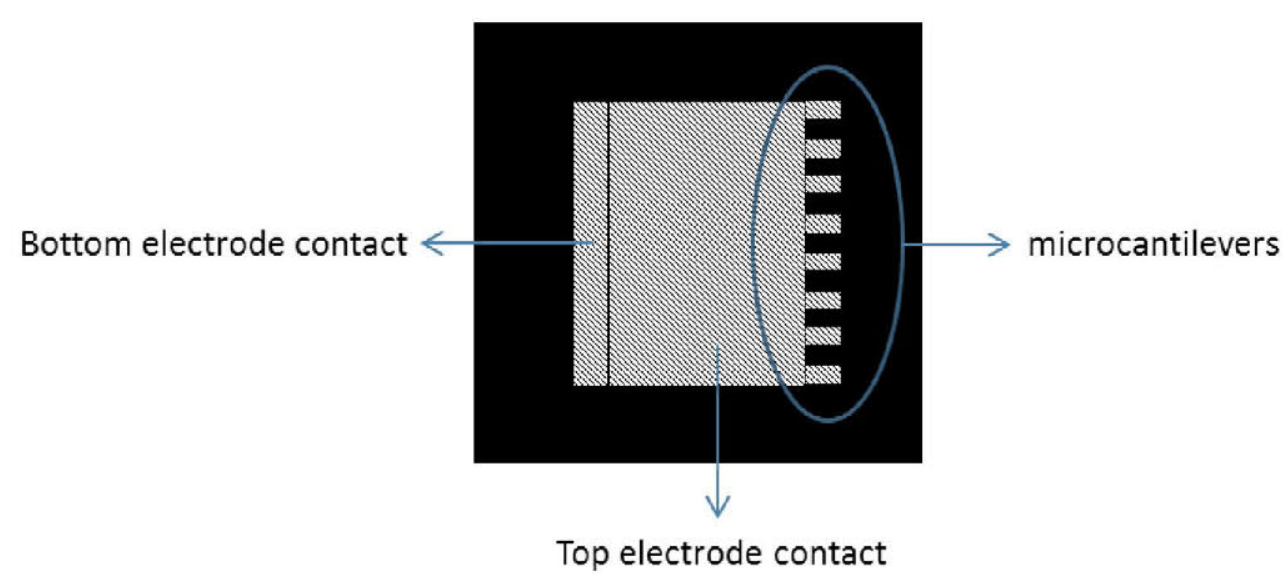

Figure 2: Schematic top view detail of the $\mathrm{Cr} / \mathrm{AlN} / \mathrm{Cr}$ structure.

The manufacturing procedure that we have used is as follows: a 4-inch, p-doped (100) silicon wafer (diced in $5 \times 10 \mathrm{~mm}^{2}$ pieces) serves as a substrate. As bottom electrode, a 0.05 to $0.2 \mu \mathrm{m}$ thick $\mathrm{Cr}$ electrode is evaporated. The sample is then covered with a 0.7 $\mu \mathrm{m}$ thick AlN piezoelectric film synthesized in a reactive sputtering process (from an aluminium target in an argon (Ar) and nitrogen $\left(\mathrm{N}_{2}\right)$ atmosphere). Since the AlN 
thickness affects the internal stress and the vibrometric behaviour (Krupa et al., 2009), for proper comparison, we have worked with just one thickness value. As top electrode, a $0.3 \mu \mathrm{m}$ thick $\mathrm{Cr}$ electrode is employed. After an optical lithography step, the whole layer set is patterned using an inductively coupling plasma dry etching process to form the microcantilever structure. The $\mathrm{Cr} / \mathrm{AlN} / \mathrm{Cr}$ microcantilever is then released via an isotropic wet etching step of the silicon substrate surface. Due to the isotropy of theetch, it exhibits equal etch rates in all directions of the silicon surface. Consequently, after the cantilevers are released, the area of cantilever clamping is also underetched, as observed in the scanning electron microscopy (SEM) analysis. This manufacturing process is the result of an optimization process which seeks maximizing the fabrication throughput while keeping it compatible with state of the art CMOS technology standards (PérezCampos, Iriarte, Hernando-Garcia, \& Calle, 2015).

To verify that the microcantilevers have been completely released from the Si surface and to determine structural and morphological properties, a SEM has been used (Oxford FEI Inspect FSO). Layer thickness measurements have been accomplished using a KLA-Tencor Alpha Step IQ profilometer. Dimensions were measured with a Nomarski microscope (Leica Leitz DMRX). The X-Ray diffraction measurements have been done using a Philips X-Pert Pro MRD diffractometer.

\subsection{Piezoelectric constant measurement}

Complementary to the piezoelectric response measured by LDV, the piezoelectric constant $d_{33}$ was also studied. Piezoelectric phenomena occur as both the direct (1) and converse (2) effect.

$$
\begin{aligned}
& P_{i}=d_{i j k} \sigma_{j k} \\
& \varepsilon_{j k}=d_{i j k} E_{i}
\end{aligned}
$$


With the direct effect (1), an external stress, $\sigma_{\mathrm{jk}}$, results in a change in polarization, $\mathrm{P}_{\mathrm{i}}$, obtaining the piezoelectric charge coefficient $d_{i j k}(i, j, k=1,2,3)$ given in coulombs per newton $\left(\mathrm{C} \mathrm{N}^{-1}\right)$. With the converse effect (2), an applied field, $\mathrm{E}_{\mathrm{i}}$, results in a strain, $\varepsilon_{\mathrm{jk}}$, obtaining the piezoelectric strain coefficient $\mathrm{d}_{\mathrm{ijk}}$, which is given in meters per volt $\left(\mathrm{m} \mathrm{V} \mathrm{V}^{-}\right.$ 1 or the equivalent $\mathrm{C} \mathrm{N}^{-1}$. The different measurement techniques to determine piezoelectric constants in materials are all based on the principle of this reversibility of the piezoelectric effect.

When the piezoelectric coefficient exhibits a positive value, the piezoelectric material expands when a positive voltage is applied to it. Conversely, it contracts when $d_{i j k}$ is negative.

Direct piezoelectric measurements are used to obtain the piezoelectric coefficient on thin film piezoelectric materials. To measure the direct piezoelectric effect, a static or quasi-static method can be used. The resonance method measurements can also be used and basically consist of determining the electrical impedance of the vibrator as a function of frequency. Although the static method is less precise than the resonance method (Newnham, 2004), the ease of use and availability of instrumentation makes it preferable. The static method employs a Berlincourt $d_{33}$ meter, for which a number of commercial systems are available. In our case, the effective values of $d_{33}$ piezoelectric coefficient were measured by means of a commercial piezo-electrical test system (PM300 by PIEZOTEST). This systems works by applying a known force to the thin film piezoelectric sample, as well as to a standard piezoelectric (commonly PZT) and comparing the resultant electric signals what enables to determine the $d_{33}$ of the sample (figure 3) (Duncan W. Bruce, Dermot O’Hare, 2011). 


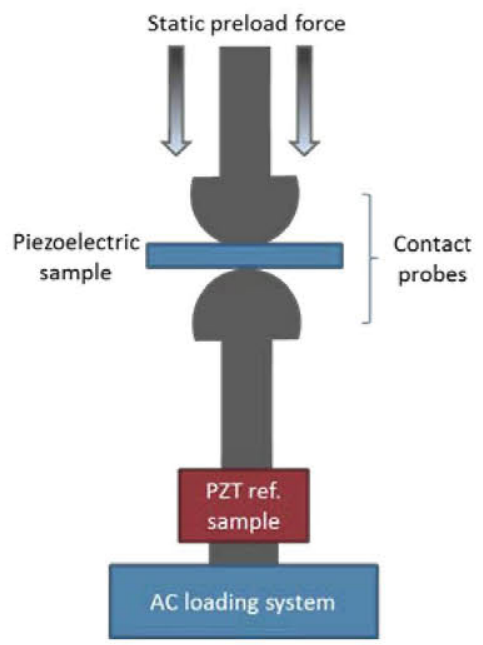

Figure 3: Schematic representation of the $d_{33}$ test system

\subsection{Simulation model}

A model based on the finite element method (FEM) was constructed using Comsol Multiphysics, a software with unique capabilities in MEMS design, modelling and simulation, using finite elements. Several authors have built models for cantilevers previously, using Comsol (Zhou, Khaliq, Tang, Ji, \& Selmic, 2005).

The elementary theory of microcantilever bending permits to obtain the resonance frequency $\omega_{\mathrm{i}}$ for a given piezoelectric biform of length $a$, width $b$ and mass density per unit of area $\rho$ and flexural rigidity $D$, the ith order natural frequency can be calculated from the following equation:

$$
\omega_{i} a^{2} \sqrt{\frac{\rho}{D}}=c_{i}
$$

where $\mathrm{c}_{\mathrm{i}}$ is a parameter which depends on the ratio $a / b$ (Javier Vázquez, Sanz, \& Sánchez-Rojas, 2007).

Material constants are provided by the Comsol Multiphysics material database. The model contains 12.757 tetrahedral elements and comprises only the suspended beam and a small part of the rest of the device, which is considered to be perfectly clamped through certain mechanical boundary conditions. Our 3D model was the basis of two 
types of calculations; on one hand, it was used to determine the natural frequencies of the modelled vibrating cantilever. At the same time, the model was also used to study the response of the cantilever when it is excited by an ac voltage applied between the electrodes, at different frequencies. The set of simulations executed allowed us to obtain valuable information about displacements, stresses and reaction charges on the surfaces.

\subsection{Laser Doppler Vibrometry}

The microcantilever vibration has been characterized by measuring the out-of-plane displacement of surfaces by laser doppler vibrometry (LDV). The electrical actuation of out-plane modes has been achieved with a specific top and bottom electrode (see Figure 1). This method measures the amplitude of the light emitted by two beams: the cantilevers and a reference one. The vibrometer works on the basis of optical interference, requiring two coherent light beams, with their respective light intensities I1 and I2, overlapping each other. The resulting intensity is not just the sum of the single intensities, but a modulated one.

LDV systems are particularly well suited for measuring vibrations where alternative methods either reach their limits or simply cannot be applied. For example, LDVs can measure vibrations up to the $1.2 \mathrm{GHz}$ range with very linear phase response and high accuracy. Measurements of the surface of liquid materials or vibrations of very small and light structures can also only be made using non-contact measurement techniques. This technique can be also used for liquid environments (J Vázquez, Rivera, Hernando, \& Sánchez-Rojas, 2009), especially useful when considering bioapplications Contacting transducers can fail when attempting to measure high amplitudes. In order to track the out-of-plane modes (natural resonance frequencies) of our AlN based microcantilevers with high precision, a scanning laser Doppler vibrometer (Polytec 
MSA-500) was used. This instrument provides a He-Ne laser spot which is focused by means of an optical microscope onto the vibrating cantilever. While scanning a grid of points on the top cantilever surface, this laser beam is scattered back through the microscope objective towards the interferometric sensor. Due to the Doppler effect, the velocity and displacement of the out-of-plane component at each grid point can be detected by either a frequency or phase modulation of the laser light. This modulation is recovered with the aid of suitable decoders. In addition to this, an animation of the movement can be simulated, taking into account the amplitude and the phase information for each of the grid points.

This optical technique was used to measure the frequencies of the resonances of the microcantilevers. The vibrometer provides the displacement of the different modes as well as their shape. The cantilevers were excited using the internal AIN actuator. Mode overview includes flexural as well as torsion modes.

The performance of the microcantilevers has been determined by applying a so called periodic chirp signal to the electrodes in a frequency range from $50 \mathrm{kHz}$ to $2 \mathrm{MHz}$. This electric stimulus consists of a superposition of sinusoidal signals designed to keep constant the amplitude in the frequency domain and hence allow for a uniform excitation. Two different decoders were used to measure at low and high frequencies, with bandwidths of $50 \mathrm{kHz}$ and $2 \mathrm{MHz}$, respectively. Electrical access to the metal electrodes was achieved by means of tips mounted in probe heads. The $\mathrm{Cr}$ top electrode was specifically designed to achieve an efficient electrical actuation of these out-ofplane modes. 


\section{Results and discussion}

In this section the experimental results obtained to study the properties of the microcantilevers and their piezoelectric behaviour as LDV measurements and WLI as well as their comparison to computer simulations are presented. Figure 4 shows some of the microcantilevers arrays fabricated and measured in this work. We focused our study on cantilevers having $50 \mu \mathrm{m}$ width and a length of $250 \mu \mathrm{m}$. The thickness of each microstructure layer was $100 \mathrm{~nm} \mathrm{Cr}$ bottom electrode/ $250 \mathrm{~nm}$ AlN/ $200 \mathrm{~nm} \mathrm{Cr}$ top electrode.

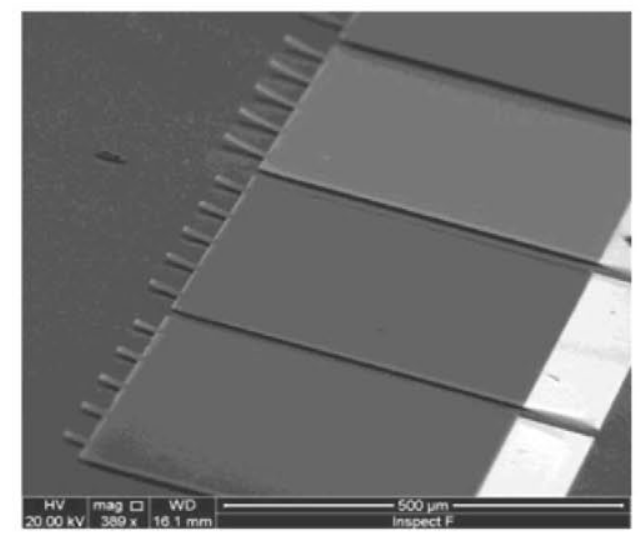

Figure 4: SEM micrographs of the microcantilever arrays studied in this work

The X-ray diffraction technique has been widely used in this work, since AlN exhibits piezoelectric properties only when the thin film is oriented in the c-axes. The width of the measured peak of a rocking curve, measured in terms of the full-width at half maximum (FWHM) has an inverse relationship with the AlN degree of c-axis orientation (Iriarte, Rodríguez, \& Calle, 2010). In this work we have obtained a small FWHM value of $2.44^{\circ}$ (figure 5), what made us expect very good piezoelectric properties, as probed later. 


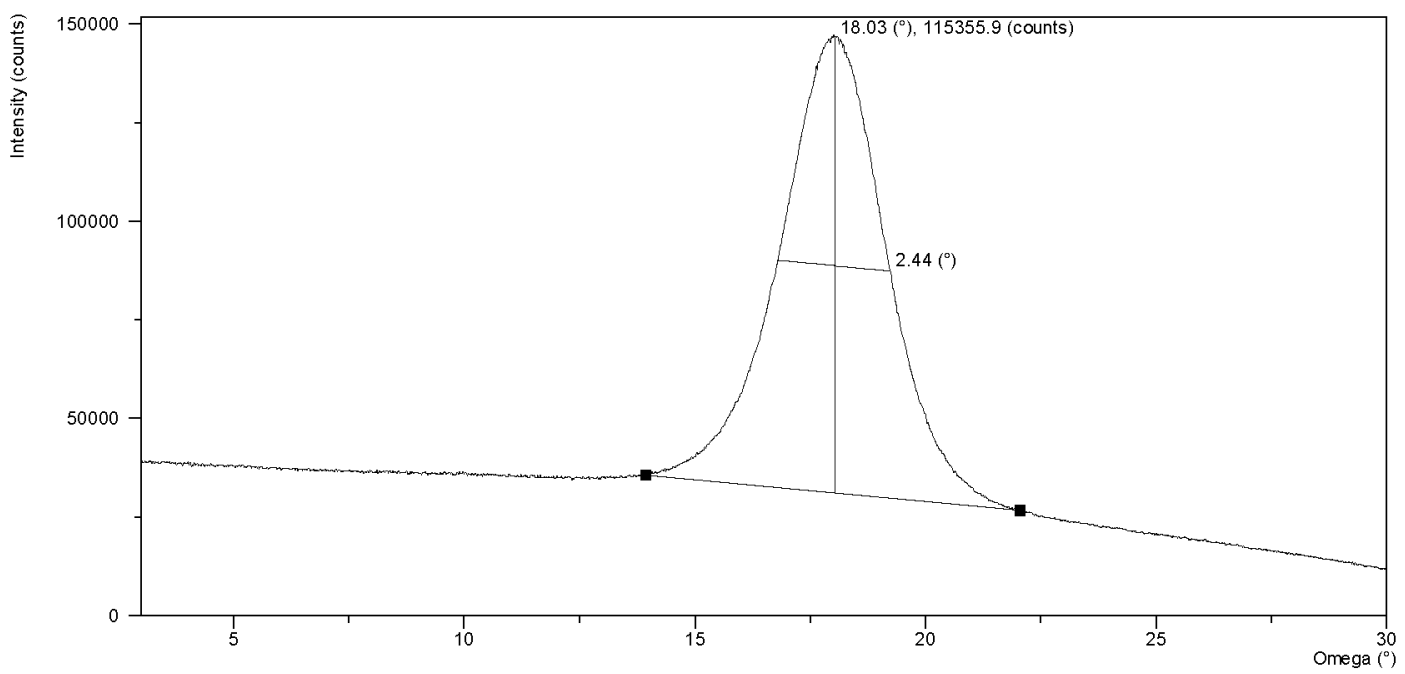

Figure 5: Rocking curve of $c$-axis oriented AlN thin films deposited at $500 \mathrm{~W}$ on $200 \mathrm{~nm}$ of Cr .

Measurements of the $d_{33}$ piezoelectric constant in $100 \mathrm{~nm} \mathrm{Cr} / 700 \mathrm{~nm} \mathrm{AlN} \mathrm{structures}$ were done, delivering a value of $-4.6 \mathrm{pC} / \mathrm{N}$. Most of piezoelectric materials achieve higher values of $\mathrm{d}_{33}\left(\mathrm{ZnO}, \mathrm{LiNbO}_{3}\right.$ and $\mathrm{LiTaO}_{3}$ above $10 \mathrm{pC} / \mathrm{N}, \mathrm{BaTiO}_{3}$ and $\mathrm{PZT}$ of 190 $\mathrm{pC} / \mathrm{N}$, whereas for PZT compositions it rises to values between 100 and $600 \mathrm{pC} / \mathrm{N}$ ) (Duncan W. Bruce, Dermot O'Hare \& Sons, 2011) (Wang et al., 2006). However, as it has been mentioned, all these materials showing better piezoelectric properties are grown at considerably higher temperatures, which increases processing cost and jeopardizes compatibility with CMOS technology. In our case, the piezoelectric constant $d_{33}$ shows a lower value, but it proves to be high enough for the microcantilevers activation and, most important, our fabrication process is compatible with standard CMOS technology and high throughput requirements. In addition, in comparison with AlN thin layers grown at higher sputtering temperatures over different substrates $\left(400^{\circ} \mathrm{C}\right)$ (Dubois \& Muralt, 2001), this parameter shows lower values than that obtained in this work, such as $3.54 \mathrm{pC} / \mathrm{N}$ over silicon, $3.4 \mathrm{pC} / \mathrm{N}$ over platinum, 1.8 $\mathrm{pC} / \mathrm{N}$ over cobalt, and $1.6 \mathrm{pC} / \mathrm{N}$ over silicon oxide. In summary, our piezoelectric material synthesis process is cheaper, faster and leads to better results. 
Computer FEM simulations were performed, providing a reasonable comparison with the experimental results. Figure 6 shows a Comsol image of the $50 \mu \mathrm{m}$ width- $250 \mu \mathrm{m}$ length microcantilever.

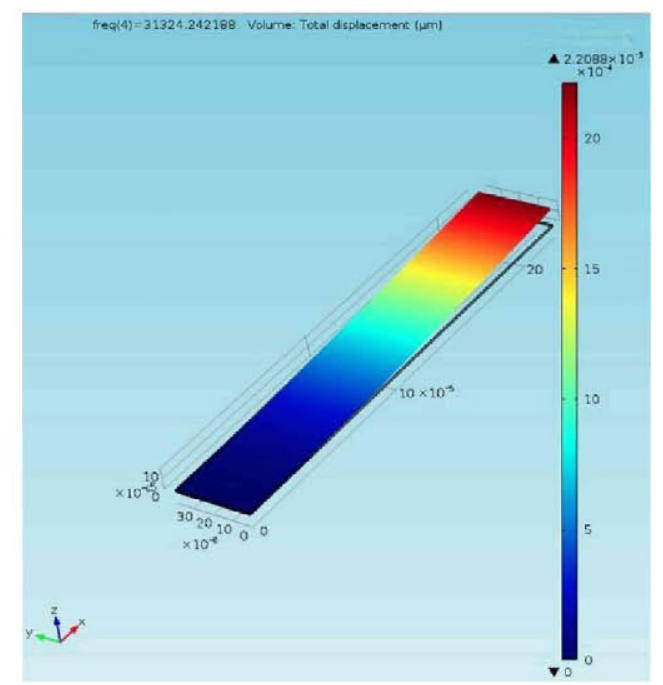

Figure 6: Comsol simulation of a microcantilever under an applied external voltage of $5 \mathrm{~V}$

In our case, the model has demonstrated to be a powerful tool to achieve a deeper understanding of the experimental results, especially considering the differences between these results and the simulation study. The cantilever model used for the simulation can be seen in Figure 7. The dashed line along the middle of the supporting silicon layer is the reference for the symmetry in the device. 


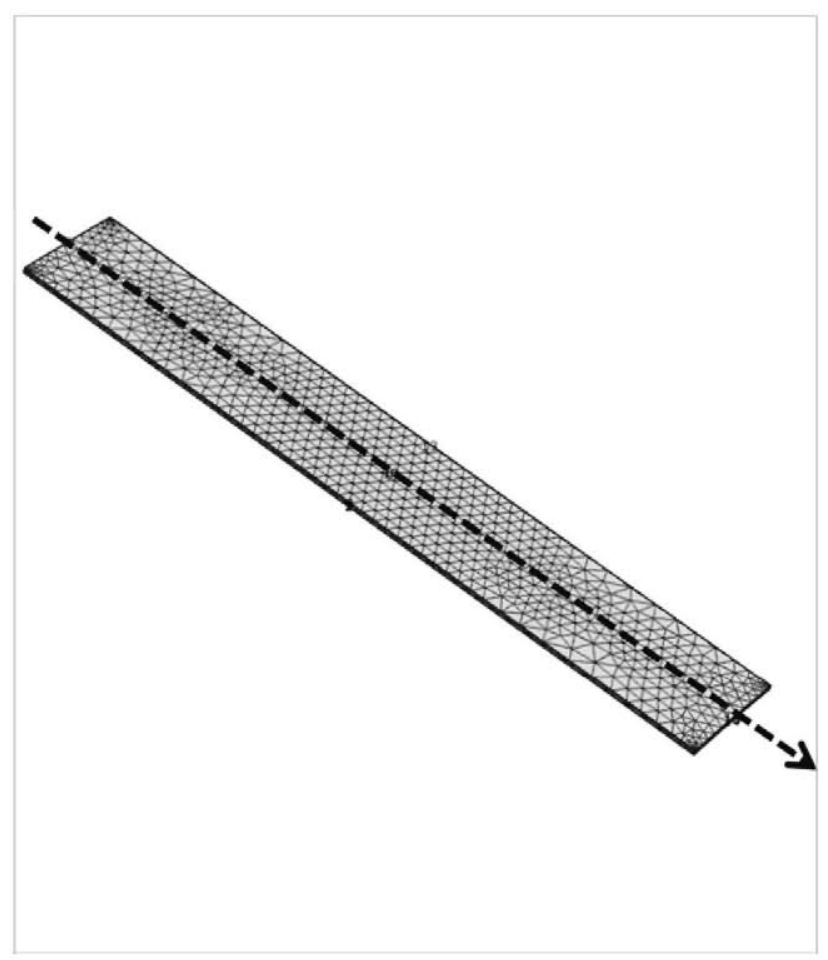

Figure 7: Schematic representation of the cantilever model used for the simulation showing its symmetry.

Table 1 shows the resonant frequencies detected by the vibrometer in the $50 \mathrm{kHz}$ to 2 $\mathrm{MHz}$ range, and the corresponding frequencies calculated by the FEM analysis. These values and their corresponding displacement are represented in figure 8.

\begin{tabular}{l|llllll}
\multicolumn{1}{c|}{ Mode } & $\mathbf{( 0 , 0 )}$ & $\mathbf{( 1 , 0 )}$ & $\mathbf{( 0 . 1 )}$ & $\mathbf{( 1 , 1 )}$ & $\mathbf{( 0 , 2 )}$ & $\mathbf{( 1 , 2 )}$ \\
\hline Laser Vibrometry (MHz) & 0.085 & 0.279 & 0.524 & 0.903 & 1.362 & 1.711 \\
Comsol (MHz) & 0.091 & 0.268 & 0.506 & 0.905 & 1.313 & 1.744
\end{tabular}

Table 1: First six frequencies peaks measured with LDV and calculated by FEM.

Table 1 shows the frequencies resonances found by LDV, and the corresponding cantilever's own eigen-frequencies deduced from FEM analysis. Figure 8 shows the images of the experimental vibration frequencies and the comparison with the finite element simulations. Different modes were detected with this system as can be seen in the figure. The WLI images show the static shape overview (Figure 8a) of the microcantilevers before being excited, or a detail of one cantilever (Figure $8 b$ ). The 
gradient (of different colours) on the beam surface indicates different height values. Both images show a slight tensile stress of the structures, but this static bowing does not affect the vibration modes significantly.

The vibration modes measured by LDV are shown in Figure 8c. The classification of the modes was made using Leissa's nomenclature (Leissa, 1969). The first number $n$ corresponds to the number of nodal lines (i.e. no vibration) along the beam, and the second one $\mathrm{m}$ is the number of nodal lines perpendicular to the beam axis. Three flexural modes $[(0,0),(0,1)$ and $(0,2)]$, and three torsional modes $[(1,0),(1,1)$ and $(1,2)]$ are clearly differentiated in Figure 8c. By observing these modes, we can see how modes $(0,1)$ and $(0,2)$ are flexural: one nodal line in case of mode $(0,1)$ and two in the case of mode $(0,2)$ perpendicular to the cantilever (represented as dashed lines of each figure $8 \mathrm{c}$ ). Mode $(1,0)$ is torsional (one nodal line appears in the centre of the cantilever parallel to it), whereas mode $(1,1)$ has both components longitudinal and torsional (two perpendicular nodal lines). Mode $(1,2)$ has also two perpendicular lines and another one parallel to the beam, showing also both longitudinal and torsional components. All the resonances detected show a symmetric displacement with respect to the dashed line of figure 7. 

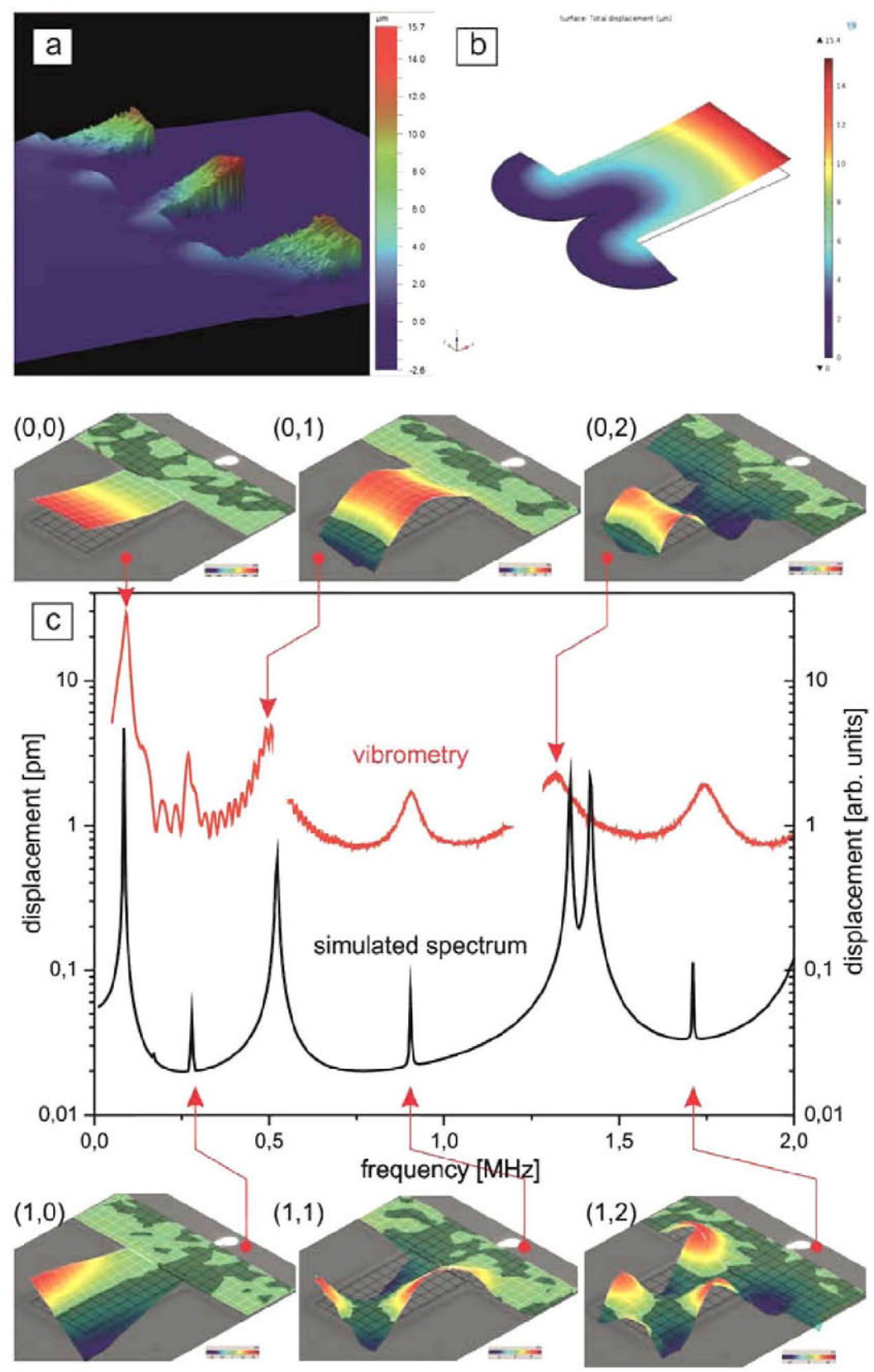

Figure 8: Vibrometry results and FEM analysis for the out of plane modal shape of the first six resonant frequencies of the cantilever.

Despite these differences, it can be asserted that the agreement between theoretical and experimental data for these six frequencies and their modal shapes studied is reasonably good. The small discrepancy found in table 1 between the FEM and the experimental frequencies for each mode has an average value of $3 \%$. This error may be attributed to a number of factors, such as the fact that the FEM calculation of the frequencies did not involve energy losses like mechanical damping. In addition, the uncertainty in the actual 
geometry of the cantilever (thickness of electrodes and AIN layer, mainly) and the deviations in the constants used for the FEM analysis might also be sources of error. The impact of underetching in the area of cantilever clamping (figure $8 \mathrm{a}$ ) due to the isotropy of the final release etching step, also affects to this variation in the frequencies values.

This manufacturing error implies also that most modes have a smaller displacement response than the simulated. It affects mostly the high-index modes, especially with torsion components. 


\section{Conclusion}

A study of the out of plane vibration response of CMOS compatible AlN-based piezoelectric cantilevers has been carried out. A laser Doppler vibrometer and a white light interferometer have been used to optically characterize the performance of the manufactured piezoelectric microcantilevers under an applied electric field. This technique permitted to analyze six resonance frequencies and their corresponding modal shapes. The high sensitivity of the vibrometer allowed us to detect both flexural modes as well as torsional modes with a symmetrical displacement referred to the line of symmetry along the cantilever.

The measured frequencies of the microcantilevers were simulated by the finite element method (FEM) using the software Comsol. FEM simulations could be compared with the eigenfrequencies of the cantilever and the corresponding modal shapes with the experimental results. Despite the small differences in the values of the resonance frequency, the experimental images precisely follow the FEM data, probing the viability of these piezoelectric microcantilevers fabricated at low temperature and compatible with CMOS technology to be implemented as both sensing as well as actuating MEMS devices. 


\section{References}

Beardslee, L. A., Addous, A. M., Demirci, K. S., Brand, O., Heinrich, S. M., \& Josse, F. (2010). Geometrical optimization of resonant cantilevers vibrating in in-plane flexural modes. In 2010 IEEE Sensors (pp. 1996-1999). IEEE. doi:10.1109/ICSENS.2010.5689930

Choi, W. J., Jeon, Y., Jeong, J.-H., Sood, R., \& Kim, S. G. (2006). Energy harvesting MEMS device based on thin film piezoelectric cantilevers. Journal of Electroceramics, 17(2-4), 543-548. doi: 10.1007/s 10832-006-6287-3

COMSOL Multiphysics ${ }^{\circledR}$ Modeling Software. (n.d.). Retrieved November 26, 2014, from http://www.comsol.com/

Doll, J. C., Petzold, B. C., Ninan, B., Mullapudi, R., \& Pruitt, B. L. (2010). Aluminum nitride on titanium for CMOS compatible piezoelectric transducers. Journal of Micromechanics and Microengineering : Structures, Devices, and Systems, 20(2), 25008. doi: $10.1088 / 0960-1317 / 20 / 2 / 025008$

Dubois, M.-A., \& Muralt, P. (2001). Stress and piezoelectric properties of aluminum nitride thin films deposited onto metal electrodes by pulsed direct current reactive sputtering. Journal of Applied Physics, 89(11), 6389. doi:10.1063/1.1359162

Duncan W. Bruce, Dermot O’Hare, R. I. W. (2011). Functional Oxides (p. 318). John Wiley \& Sons.

Duncan W. Bruce, Dermot O'Hare, R. I. W., \& Sons, J. W. \&. (2011). Functional Oxides (p. 318). John Wiley \& Sons.

Franke, A. E., Bilic, D., Chang, D. T., Jones, P. T., King, T.-J., Howe, R. T., \& Johnson, G. C. (1999a). Post-CMOS integration of germanium microstructures. In Technical Digest. IEEE International MEMS 99 Conference. Twelfth IEEE International Conference on Micro Electro Mechanical Systems (Cat. No.99CH36291) (pp. 630-637). IEEE. doi:10.1109/MEMSYS.1999.746901

Franke, A. E., Bilic, D., Chang, D. T., Jones, P. T., King, T.-J., Howe, R. T., \& Johnson, G. C. (1999b). Post-CMOS integration of germanium microstructures. In Technical Digest. IEEE International MEMS 99 Conference. Twelfth IEEE International Conference on Micro Electro Mechanical Systems (Cat. No.99CH36291) (pp. 630-637). IEEE. doi:10.1109/MEMSYS.1999.746901

Iriarte, G. F. (2003). Surface acoustic wave propagation characteristics of aluminum nitride thin films grown on polycrystalline diamond. Journal of Applied Physics, 93(12), 9604. doi: $10.1063 / 1.1574172$

Iriarte, G. F., Rodríguez, J. G., \& Calle, F. (2010). Synthesis of c-axis oriented AIN thin films on different substrates: A review. Materials Research Bulletin, 45(9), 1039-1045. doi:10.1016/j.materresbull.2010.05.035

Krupa, K., Józwik, M., Gorecki, C., Andrei, A., Nieradko, Ł., Delobelle, P., \& Hirsinger, L. (2009). Static and dynamic characterization of AlN-driven microcantilevers using optical interference microscopy. Optics and Lasers in Engineering, 47(2), 211-216. doi:10.1016/j.optlaseng.2008.05.001

Lachut, M., \& Sader, J. (2007). Effect of Surface Stress on the Stiffness of Cantilever Plates. Physical Review Letters, 99(20), 206102. doi:10.1103/PhysRevLett.99.206102

Leissa, A. W. (1969). Vibration of plates. Retrieved from http://ntrs.nasa.gov

Lu, Z., Hiskes, R., DiCarolis, S. A., Route, R. K., Feigelson, R. S., Leplingard, F., \& Fouquet, J. E. (2011). Epitaxial LiNbO3 thin films on sapphire substrates grown by solid source MOCVD. Journal of Materials Research, 9(09), 2258-2263. doi:10.1557/JMR.1994.2258

Newnham, R. E. (2004). Properties of Materials: Anisotropy, Symmetry, Structure. (O. Oxford, Ed.) (p. 392).

Oliver, M. J., Hernando-García, J., Ababneh, A., Seidel, H., Schmid, U., Andrés, J. P., ... Sánchez-Rojas, J. L. (2012). Resonantly excited AlN-based microcantilevers for immunosensing. Microsystem Technologies, 18(7-8), 1089-1094. doi:10.1007/s00542012-1444-x 
P. Gr. Lucuta, Fl. Constantinescu, D. B. (1985). Structural Dependence on Sintering Temperature of Lead Zirconate-Titanate Solid Solutions. Journal of the American Ceramic Society, 68(10), 533-537. doi:10.1111/j.1151-2916.1985.tb11519.x

Pei, J., Tian, F., \& Thundat, T. (2004). Glucose biosensor based on the microcantilever. Analytical Chemistry, 76(2), 292-7. doi:10.1021/ac035048k

Pérez-Campos, A., Iriarte, G. F., Hernando-Garcia, J., \& Calle, F. (2015). Post-CMOS compatible high-throughput fabrication of AlN-based piezoelectric microcantilevers. Journal of Micromechanics and Microengineering, 25(2), 025003. doi:10.1088/0960$1317 / 25 / 2 / 025003$

Ruiz, V., Hernando-García, J., Ababneh, a., Seidel, H., Schmid, U., \& Sánchez-Rojas, J. L. (2012). Modelling and characterization of AlN-actuated microcantilevers vibrating in the first in-plane mode. Microsystem Technologies, 18(7-8), 997-1001. doi:10.1007/s00542012-1428-x

Vashist, S. K. (2007). A Review of Microcantilevers for Sensing Applications. Journal of Nanotechnology, 3, 1. doi:10.2240/azojono0115

Vázquez, J., Rivera, M. A., Hernando, J., \& Sánchez-Rojas, J. L. (2009). Dynamic response of low aspect ratio piezoelectric microcantilevers actuated in different liquid environments. Journal of Micromechanics and Microengineering, 19(1), 015020. doi:10.1088/0960$1317 / 19 / 1 / 015020$

Vázquez, J., Sanz, P., \& Sánchez-Rojas, J. L. (2007). Behaviour of forbidden modes in the impedance characterization and modeling of piezoelectric microcantilevers. Sensors and Actuators A: Physical, 136(1), 417-425. doi:10.1016/j.sna.2006.11.005

Wang, X. B., Song, C., Li, D. M., Geng, K. W., Zeng, F., \& Pan, F. (2006). The influence of different doping elements on microstructure, piezoelectric coefficient and resistivity of sputtered ZnO film. Applied Surface Science, 253(3), 1639-1643. doi:10.1016/j.apsusc.2006.02.059

Wu, G., Ji, H., Hansen, K., Thundat, T., Datar, R., Cote, R., .. Majumdar, A. (2001). Origin of nanomechanical cantilever motion generated from biomolecular interactions. Proceedings of the National Academy of Sciences, 98(4), 1560-1564. doi:10.1073/pnas.98.4.1560

Yahaya, M., \& Salleh, M. M. (2004). Bismuth titanate thin film for pressure sensor prepared by sol gel method. In 2004 IEEE International Conference on Semiconductor Electronics (p. 4 pp.). IEEE. doi:10.1109/SMELEC.2004.1620957

Zenin, A. A., Lysikov, S. V., Nersesyan, M. D., Kuznetsov, G. P., \& Merzhanov, A. G. (1993). Synthesis of LiTaO3 in self-propagating high-temperature wave. Combustion, Explosion, and Shock Waves, 29(1), 57-61. doi:10.1007/BF00755330

Zhou, W., Khaliq, A., Tang, Y., Ji, H., \& Selmic, R. R. (2005). Simulation and design of piezoelectric microcantilever chemical sensors. Sensors and Actuators A: Physical, 125(1), 69-75. doi:10.1016/j.sna.2005.07.009

\section{Acknowledgments}

This work has been supported by Spanish Ministerio de Educacion y Ciencia under project no. TEC2010-19511. 